\title{
Parallel Ripple Search - Scalable and Efficient Pathfinding for Multi-core Architectures
}

\author{
Sandy Brand and Rafael Bidarra \\ Delft University of Technology \\ Mekelweg 4, 2628 CD Delft, Netherlands \\ sjrbrandegmail.com, R.Bidarra@tudelft.nl \\ http://graphics.tudelft.nl
}

\begin{abstract}
Game developers are often faced with very demanding requirements on huge numbers of agents moving naturally through increasingly large and detailed virtual worlds. With the advent of multi-core architectures, new approaches to accelerate expensive pathfinding operations are worth being investigated. Traditional single-processor pathfinding strategies, such as $\mathrm{A}^{*}$ and its derivatives, have been long praised for their flexibility. We implemented several parallel versions of such algorithms to analyze their intrinsic behavior, concluding that they either have a large overhead, yield far from optimal paths, do not scale up to many cores, or are cache unfriendly. In this paper we propose Parallel Ripple Search, a novel parallel pathfinding algorithm that largely solves these limitations. It utilizes a high-level graph to assign local search areas to CPU cores at 'equidistant' intervals. These cores then use A* flooding behavior to expand towards each other, yielding good 'guesstimate points' at border touch on. The process does not rely on expensive parallel programming synchronization locks, but instead relies on the opportunistic use of node collisions among cooperating cores, exploiting the multi-core's shared memory architecture. As a result, all cores effectively run at full speed until enough waypoints are found. We show that this approach is a fast, practical and scalable solution, and that it flexibly handles dynamic obstacles in a natural way.
\end{abstract}

\section{Introduction and Previous Work}

As virtual game worlds grow increasingly larger, pathfinding has once again come into the spotlight. The basic motivation for this is that, being a computationally expensive but indispensable component in many games, any performance gains here will typically bring about noticeable improvements. In this line, more attention is currently being paid to re-designing pathfinding algorithms, so that they better suit current multi-core architectures.

Classic pathfinding algorithms such as $A^{*}$ and its many derivatives, have been long praised by game developers for their flexibility and completeness. $\mathrm{A}^{*}$ is a best-first search strategy that relies on a cost computing function $f(n)=g(n)+h(n)$ for providing rough cost estimations of a path running through a node $n$ of a search graph (see [10]). Function $g(n)$ represents the currently known cost for reaching node $n$ from the start node $S$, and heuristic estimation function $h(n)$ is often implemented by using a 
cheap 'guesstimate' of the remaining travel distance, such as a Manhattan or Euclidean distance, between node $n$ and the goal node $G$. This heuristic function effectively controls how $\mathrm{A}^{*}$ floods its search space. Moreover, $h(n)$ results in optimal paths as long as it remains 'admissible', i.e. it never overestimates the true cost for an actual path between node $n$ and the goal node $G$.

The $\mathrm{A}^{*}$ algorithm utilizes the $f(n)$ function to maintain a sorted Open list of most promising search candidates while it iterates through the search space, which is also its most computationally expensive component. For each iteration, the algorithm will remove the most promising candidate and place on the list all its not yet visited neighbors. If a neighbor node was already in the Open list, A* will perform a crucial 'correction step': it determines if a cheaper path was possible through the candidate node and, if so, modifies its entry accordingly in the Open list.

As the flood boundary grows, the algorithm takes increasingly more time to find each successive node that forms the desired path. Empirically, the node which is halfway down the resulting path is closed at roughly a third of the total time taken to find the complete path. This sorting component has also proven a road-block for parallelization attempts because it institutes a data dependency that would generate a lot of communication overhead on distributed processing architectures. Many past attempts have already been made to eliminate this need for sorting on single processor architectures such as Iterative Deepening $A$ * (or IDA* for short) (see [9]), but have not resulted in easier distributed processing schemes.

A late addition to the family of $A^{*}$ derivatives, which we used extensively in this research, is called Fringe Search (FS); see [2]. Fringe Search avoids the need for a sorted candidate list by simply keeping track of all nodes at its search boundary (or 'fringe') and opening those that are less expensive than a certain threshold value, which is iteratively incremented. Although this now forces the algorithm to scan through a large unsorted Now list from begin to end, it gains its speed-up by making the actual visitations extremely cheap. Each node that has an $f(n)$ value higher than the threshold value will simply be moved to a 'Later' list. Each new iteration starts by simply swapping the Now and Later lists, and the node/list manipulations themselves can be implemented very effectively using simple pointer logic.

Recent path finder parallelization attempts (see [3] and [6]) have mainly focused on translating $\mathrm{A}^{*}$ variants into shaders, so that they can run on GPUs or similar Vector Processors. These schemes benefit from either taking work-load off the main CPU, or by running pathfinders for a large amount of agents in parallel. Although such approaches have been very successful, these also have the drawback that they take up precious resources that one would rather devote solely to rendering graphics. Also, from a practical point of view, a game-play programmer will have to take extensive measures to apply such pathfinding approaches without serious disruptions of the rendering pipeline. For multi-core architectures, specifically, the world simulation and rendering logic are often running in separate threads that are uniquely assigned to specific cores.

A more traditional approach has been to parallelize $A^{*}$ and related algorithms, and make them more suitable for distributed computing on CPU clusters and grids (see [7], [8] and [12]). Although these attempts have demonstrated beneficial advantages, they also require more 'exotic' hardware and software approaches such as MPI (Message Passing Interface) which are highly uncommon on virtually any gaming 
hardware platform up to this date. For practical purposes, these approaches are thus unsuitable and have currently no real relevance in the game development industry.

In conclusion, there is a definite need for simple and portable variants of the $\mathrm{A}^{*}$ algorithm that successfully and efficiently exploit today's multi-core architectures. This paper first describes and compares a number of parallel pathfinding implementations, focusing on the efficiency of their multi-core use. They all rely on the underlying hardware to implicitly perform the necessary synchronizations, without any blocking. We then introduce a novel algorithm called Parallel Ripple Search, that can easily scale with the number of available CPU cores. It requires no special libraries or hardware interfacing, nor any special synchronization primitives.

\section{Parallel Pathfinding Implementations}

In this section we describe our investigation on a number of parallelized variants of $A^{*}$ and Fringe Search, in order to study how they perform on multi-core architectures. Please refer to [5] for a detailed discussion of each algorithm, including its pseudocode. This study gave us significant insight on how to effectively utilize the computing potential of these architectures for pathfinding purposes.

\subsection{PBS: Parallel Bidirectional Search}

The most obvious strategy to use two CPU cores for pathfinding is to have them start at each path extremity, search towards each other and let them 'meet halfway'; hence the name Parallel Bidirectional Search (PBS). As the main strategy of A* is to keep opening the most promising node, we can consider all nodes at the boundary of the flood area 'most' optimal (although this is not always strictly true). Whenever we hit a node flooded by an opposite core, we can immediately complete the path using the alternate core's pathfinding meta data. There is no need for expensive mutexes; both cores can just check a shared 'break flag' in main memory to see if they should stop because the other core found a collision or gave up.

An example path found using PBS is shown in Figure 1. On the left, we see that a collision was detected somewhere halfway, when both cores have done, in parallel, virtually the same amount of work. Connecting the two 'half-paths' together yields a path that is only slightly more expensive than the most optimal path. Most discrepancies relative to $A^{*}$ are not erroneous when taking the flow of the full path into account, but rather the result of a different bias because of the reversed search direction. The insignificant loss of optimality is due to the fact that PBS stops the search just a bit too soon, when it detects a collision. Often, the area around the collision node does not get fully flooded, so potentially there might be cheaper nodes in this area, which will no longer be discovered. However, this deviation is generally very low given a fairly uniform travel cost between neighboring nodes.

We can, therefore, conclude that, strictly speaking, PBS is no longer optimal, but it is still complete, i.e. it will find a path if it exists. In worst-case scenarios where no collision occurs, PBS is basically reduced to a normal $\mathrm{A}^{*}$ search. 


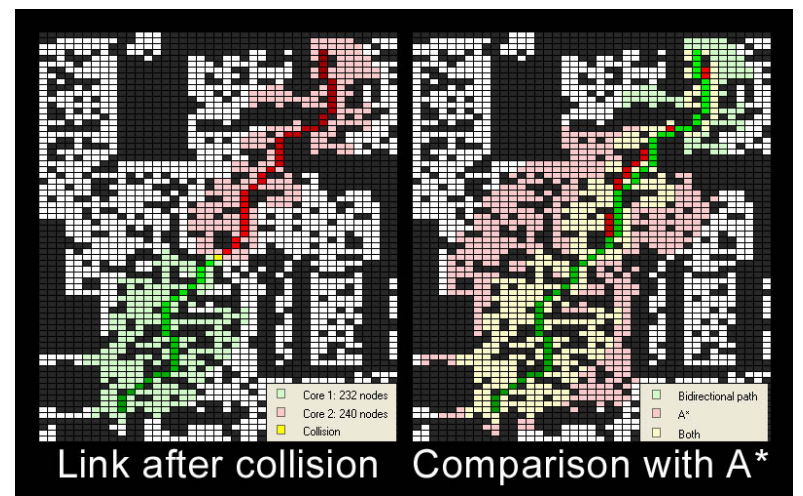

Fig. 1. (Left) Using PBS, two cores flood towards each other until a collision is detected. The full path is then constructed by linking both 'halves' together at the collision node. (Right) PBS path overlaid with the optimal A* path, whereby the red cells denote path deviations. Clearly, much less nodes are flooded by PBS than by A*.

\subsection{DFS: Distributed Fringe Search}

Our second attempt towards parallelization speed-up was to use the Fringe Search's Now and Later lists to literally distribute 'work' among multiple CPU cores. As mentioned above, during each pass through the Now list, Fringe Search performs some very simple tests in order to determine if new nodes should be processed. This processing mostly involves adding new nodes to the Later list. The main idea behind the new algorithm we came to call Distributed Fringe Search (DFS), is thus to distribute the Now List over all available cores, have them process their share, merge the individual Later lists, swap this with Now, and start all over again. A nice feature of Fringe Search is that the Now and Later lists do not need any sorting, thus distributing them is very easy. Also, each core can compute the smallest $f(n)$ value it has found locally, so that the master core can collect them and only needs to do a few comparisons to determine the cost threshold that should be used next for all cores.

The main advantage of DFS is that it can utilize more cores effectively, being no longer limited to two cores as PBS. Although this is a great strength, it is also its Achilles' heel. By distributing nodes 'arbitrarily' over multiple cores, we have lost the ability to perform the 'correction step' as a normal $A^{*}$ and/or Fringe Search implementation would do. This means that it is no longer guaranteed that DFS will find the cheapest path, i.e. DFS is not optimal, as we can clearly see in Figure 2.

Another drawback of DFS is that it is also no longer possible to keep track of the 'parent-child' relation of nodes during flooding, although this information is needed to reconstruct the resulting path. The solution, therefore, is to use a shared buffer in which all cores write to signal flooded nodes to each other, and then a separate 'private' buffer for reach core to store $g(n)$ values to evade race-conditions. The final path can then be correctly reconstructed by starting at the goal node $G$ and then searching our way back to the start node $S$ by repeatedly traversing towards the neighboring node with the lowest $g(n)$ value found in any of the private buffers. Although this sounds discouraging, it is, in practice, not critical; we found surprisingly 


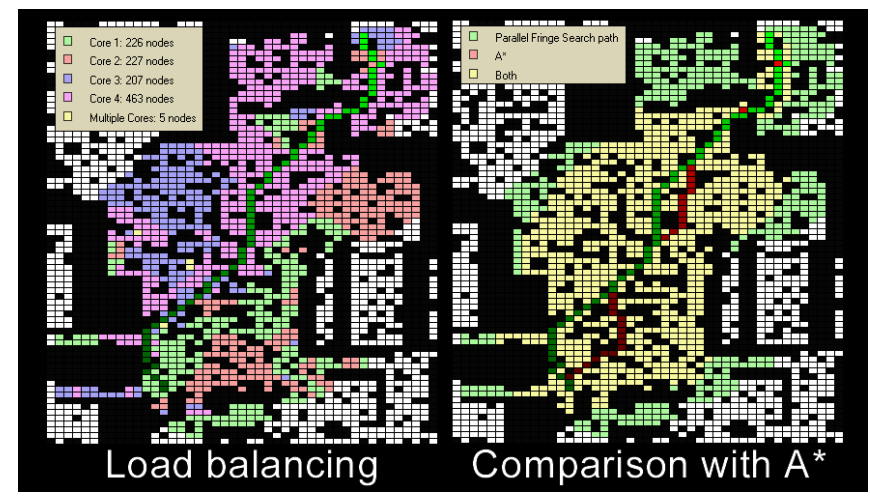

Fig. 2. (Left) The load-balance achieved by DFS. (Right) With the loss of the corrective property, DFS ends up with less than optimal paths. Note that the deviations with the optimal A* path are also just a matter of different bias, the actual additional path cost was only roughly $2 \%$.

few cases of 'over-flooding' (nodes tagged 'Multiple Cores', in Figure 2, left). Mostly, nodes only have a single $g(n)$ computed for them, so no expensive floating-point comparisons are needed to find the lowest one.

The overall load-balance seems fairly good (see Figure 2, left), although we have noticed that there is always one core that seems to be doing most of the work. That core has often flooded most of the areas in which the final path was found, suggesting that this is likely due to the $\mathrm{A}^{*}$ heuristic function: this function is designed to pull the search towards the goal node and, as long as this goes on 'unhindered', it will always favor nodes for that particular core. The other cores will often be searching through 'branches' elsewhere that later on turn out to be dead-ends.

Conceptually, the DFS approach sounds promising because it allows us to distribute the workload quite naturally over all available cores. In practice, however, the results obtained are less spectacular. After some profiling, it turned out that a significant amount of time is still wasted on cores waiting for each other, suggesting that the load balancing is still far from optimal.

\subsection{PHS: Parallel Hierarchic Search}

Another attractive way to utilize multi-core architectures is to have each core find small segments of the total path. Small searches whereby the segment's goal node is relatively close to its start node are significantly faster because far less nodes will become flooded. In order to do this, however, we need to guess where some waypoints will be located in the search space so that, as it were, we can 'connect the dots' between them. This can only be properly done if we employ a high-level graph representation of the actual graph to search through. With this high-level graph we can roughly guess how the full path will traverse the search space and obtain way-points from it; hence the name Parallel Hierarchic Search (PHS).

There are many techniques to obtain such high-level graphs, ranging from manually adding way-points to automated schemes such as 'Probabilistic Roadmap Method'; see [1], [2] and [11]. For our PHS implementation, we created a grid randomization 


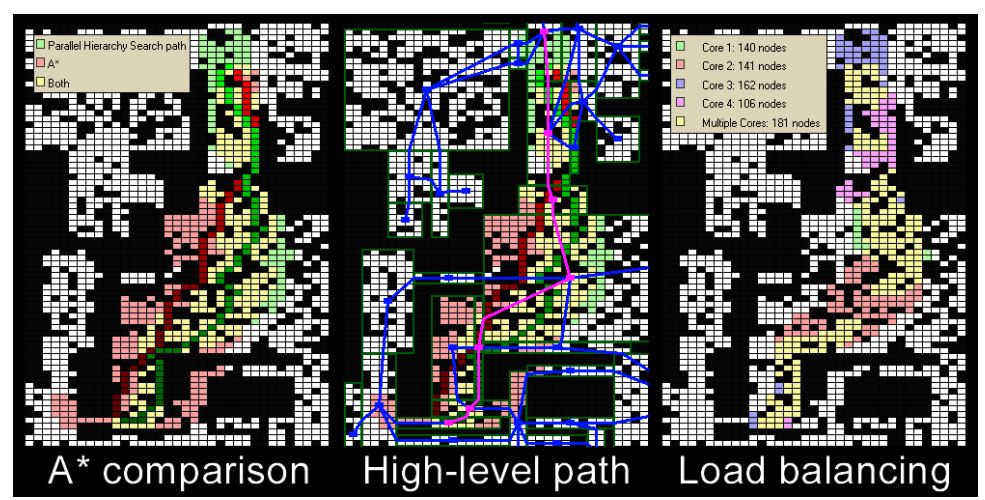

Fig. 3. A path generated by the PHS algorithm. (Left) Substantially less nodes have been flooded than with classic $\mathrm{A}^{*}$, but the resulting path is not as optimal and smooth. (Middle) The highlevel graph and path used to form the resulting path. (Right) The flooding progress per core.

algorithm with a top-down approach, which generates 'chambers' that are linked with smaller corridors, which are then filled with randomly placed obstacles. This enabled us to easily generate lots of correct high-level hierarchies so that we could run large test batches.

Each node in the high-level graph is linked to a corresponding anchor node in the actual graph that needs to be searched. The first step in the PHS algorithm is to find a path through the high-level graph and then finding sub-paths connecting the consecutive anchor nodes. Doing this will, however, never result in an immediately natural looking path because the anchor nodes might be needlessly off course. So a 'beautification' step is applied by constructing new way-points halfway at the found path segments (by just picking the middle node of the path-segments sequence of solution nodes). The idea behind this is that it will help us find 'short-cuts' between the high-level way-point anchor nodes. We found that just a single beautification iteration already yields quite acceptable results.

Parallelizing the algorithm is basically a matter of having the master core generate the way-points, and then letting all the cores try to construct the path segments. To enable the cores to gain access to the path segments information buffer, we are forced to employ a more expensive 'critical section' which is provided by the operating system. This will allow safe access to selecting a path segment and returning a pointer to found path segment solutions (cores are not allowed to clear these solutions until all processing has been completed, so that the master core can safely access them). We can give each core its own copy of the graph so that there will be no cache collisions during the searches themselves.

Figure 3 shows an example of a path generated using the PHS algorithm. We can clearly see that PHS only floods nodes in the near vicinity of the final path and does not fan out into a 'leaf' shaped flood space as $\mathrm{A}^{*}$ would do. The algorithm was about 1.6 times faster than a classic $\mathrm{A}^{*}$ approach, but this came with a penalty: the resulting path has a noticeably higher cost, and has a less 'smooth' appearance. In the middle of the figure we can see that the high-level path is distanced quite far away from the optimal path, as could be expected. Finally, on the right we see that the load balancing 
is fairly acceptable. A lot of nodes have been flooded by multiple cores, but this is again due to the second phase smoothing. Because cores will process new path segments when they are done with the previous one, we will see some nodes being flooded by different cores in different phases.

In general, the results obtained with PHS are rather divergent. In some cases we can obtain very good speed-ups, and in other cases we do not. The overall path quality leaves a lot to be desired; often the paths will stray quite a bit from the optimal path, further contributing to increase the search duration.

\section{Algorithm Evaluation and Comparison}

In all preceding experiments, no special attempts have been made to 'optimize' the parallel algorithms, other than those dictated by common sense. Moreover, they all use established basic libraries, such as the C++ Standard Template Library (STL), expressly chosen for their stability rather than for their performance.

We obtained our measurements from a total of 2000 samples, by finding 100 random paths in 20 random maps. For each sample, we measured the time taken to find a path between 2 randomly selected nodes from a 400 by 4008 -way connected uniform grid, internally represented by a directed graph. An Euclidean distance was used as A* search heuristic. Each sample was repeated 5 times for each algorithm so that cache content would 'stabilize'. The best result of all the taken samples was then taken as the ultimate measurement result. All threads and processes were running on highest priorities. All samples have been taken on a $2.4 \mathrm{GHz}$ Intel Core2 Quad CPU running Windows XP Pro SP2.

Because Fringe Search plays such a prevalent role in our experiments, wherever possible we have used this algorithm as an $A^{*}$ alternative. Therefore, Fringe Search has also been included in all measurements so that we can clearly tell if the speed-up is due to the parallelization, and not just the fact that Fringe Search was used instead of a classic $\mathrm{A}^{*}$ implementation.

The measurement results are shown in Figure 4. For very short paths the results are mixed, which probably means that the parallelization overhead is too high compared to the amount of work that has to be done. As the path length increases, the parallelized algorithms start to outperform the classic A* implementation extensively. Still, PHS is the 'main loser', as it has the worst overall performance, probably due to requiring a mutex and 'beautification' iteration. It has also by far the worst path cost overhead, ranging from $20 \%$ up to $45 \%$. For DFS, we can conclude that it is only worthwhile on longer paths; otherwise, the normal Fringe Search implementation still (slightly) outperforms it. It is apparently only for the longer paths that cores manage to get work done without interfering too much with each other's caches, which makes sense because the flooded areas will be much larger and further spaced apart. DFS can yield up to 2,2 speed-up relative to a classic A* implementation. As expected, its qualitative output is hampered by the fact that DFS has lost its corrective property in a far more significant degree than that of PBS: it has up to $4 \%$ additional path cost. For PBS, the loss of its corrective property is only accumulated near the area of the collision node, which is generally very small. This clearly makes PBS a winner on all fronts: up to an impressive speed-up of 6,7 relative to $A^{*}$, while generating paths that, on average, are less than $1 \%$ more expensive than the optimal $\mathrm{A} *$ path. 

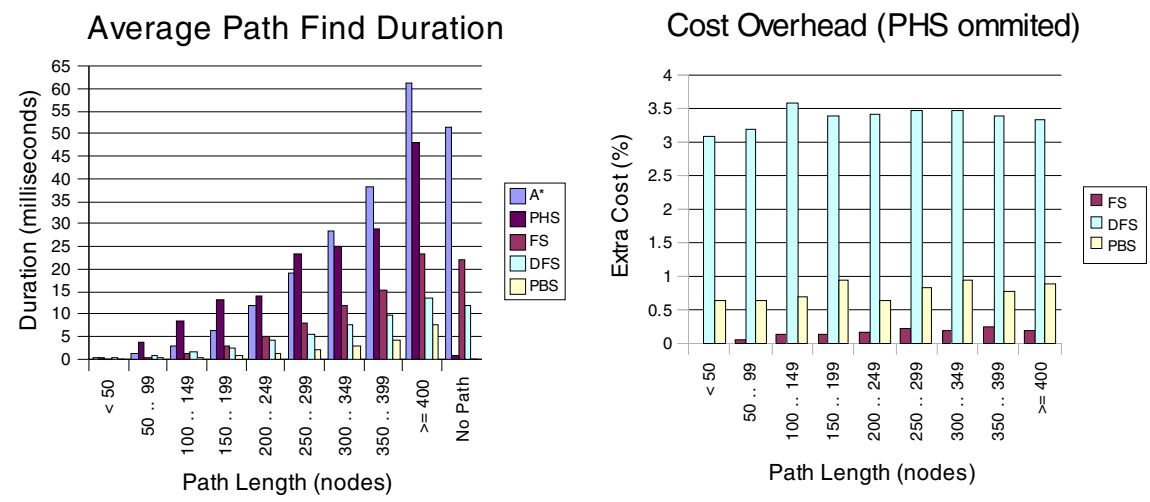

Fig. 4. (Left) Overview of average pathfinding duration. Note how PBS can outperform a classic A* implementation almost 7 times. (Right) Overview of average path cost overhead, relative to the length of the optimal path found by classic A*. (PHS results are omitted, as they can raise up to $45 \%$ ).

The analysis of all these results is summarized in Table 1. We conclude that:

1. Cache penalties have by far the largest impact on these algorithms' performance on multi-core architectures. The longer we can prevent cores to flood nodes in each other's areas, the better the performance will be. PBS clearly does this best because both cores start their search at the maximum possible distance apart from each other.

2. High-level graphs tend to 'malform' paths interpolated from them, and require quite some 'post-processing' to smoothen them out.

3. High-level paths can seriously thwart the path finder when dynamic obstacles are in the way.

\section{PRS: Parallel Ripple Search}

In this section, we present a novel algorithm called Parallel Ripple Search (PRS), which capitalizes on the results of the experiments above, in order to combine the strengths of all those algorithms while minimizing their weaknesses.

\subsection{Algorithm}

Parallel Ripple Search requires a high-level graph to guess where the final path will be located in the search space, and uses it to position the cores at roughly equidistant way-points. However, in contrast to PHS, the cores will now find path segments by doing a normal $\mathrm{A}^{*}$-like flood towards their nearest neighbors instead. Like ripples in a pond, at some point their flood boundaries will overlap and we can use these collisions to link the path-segments together into the full path.

The high potential of PRS is that when we find enough collisions, we can 'shortcircuit' and find connecting paths through previously flooded areas (for which we know that a path must exist). There is a good chance that these areas might still be 
Table 1. Summary of strengths and weaknesses of the 3 parallel pathfinding implementations

\begin{tabular}{|c|c|c|c|}
\hline & $\begin{array}{l}\text { PBS: } \\
\text { Parallel Bidirectional } \\
\text { Search }\end{array}$ & $\begin{array}{l}\text { DFS: } \\
\text { Distributed Fringe } \\
\text { Search }\end{array}$ & $\begin{array}{l}\text { PHS: } \\
\text { Parallel Hierarchy } \\
\text { Search }\end{array}$ \\
\hline Speed increase over classic $A^{*}$ & $2,5-6,7$ & $1,6-2,2$ & $1,2-1,6$ \\
\hline Path cost overhead & $<1 \%$ & $3 \%-4 \%$ & $20 \%-45 \%$ \\
\hline Scalability & Bad: 2 cores only & 2 or more & $\begin{array}{l}2 \text { or more (but } \\
\text { potentially much more } \\
\text { effective than DFS) }\end{array}$ \\
\hline Extra memory required & Low & High & Medium \\
\hline Load-balancing & $\begin{array}{l}\text { Very easy: both cores } \\
\text { always run at full } \\
\text { speed }\end{array}$ & $\begin{array}{l}\text { Hard, so we are } \\
\text { always bound by the } \\
\text { slowest CPU core }\end{array}$ & $\begin{array}{l}\text { Medium, the quality } \\
\text { of the high-level } \\
\text { graph will } \\
\text { automatically improve } \\
\text { this }\end{array}$ \\
\hline Cache 'friendliness' & Very friendly & Very unfriendly & Fairly friendly \\
\hline Implementation & $\begin{array}{l}\text { Very easy and } \\
\text { intuitive }\end{array}$ & $\begin{array}{l}\text { More involved, needs } \\
\text { many more } \\
\text { synchronization } \\
\text { moments, requires } \\
\text { special techniques to } \\
\text { optimize }\end{array}$ & $\begin{array}{l}\text { Fairly easy (closer to } \\
\text { 'classic' } \\
\text { parallelization). }\end{array}$ \\
\hline Other & $\begin{array}{l}\text { Any A* variant can be } \\
\text { used }\end{array}$ & - & $\begin{array}{l}\text { Requires high-level } \\
\text { graph }\end{array}$ \\
\hline
\end{tabular}

(partially) lingering in a core's cache, so that accesses can be fast. This algorithm is also able to deal with dynamic obstacles a lot better than PHS could. If way-points turn out to be (partially) blocked, then this will just mean that adjacent ripples will not collide (not now, at least). So although it might take longer before a collision occurs with a ripple located further away, we will no longer run the risk of pulling the path in weird directions. Another advantage of this new approach is that we can utilize many more cores; basically one for each segment of the high-level path, thus overcoming the main restriction of PBS.

The cores that flood from the start node $S$ and goal node $G$ process what we could call the 'essential' ripples: we call them the essential cores, as opposed to all other non-essential cores. In a worst-case scenario whereby none of the non-essential ripples ever collide with the essential ones, PRS will basically have degraded to a parallel bidirectional search (PBS), which was shown to perform very well.

The entire algorithm is roughly described by the following steps:

- Find a high-level path $P$ between start node $S$ and goal node $G$.

- Two cores are assigned to the way-points at both ends (S and G) of path $P$.

- Depending on the number of edges in path $P$ we try to assign other cores at fairly equidistant way-points, these cores will form the 'non-essential ripples'.

- Phase 1: All cores start flooding the search space until enough collisions have been found to form a complete path: 
- Essential cores search towards each other's local start node (basically like PBS).

- The remaining non-essential cores search towards the local start nodes of their direct neighbors.

- The master core will examine all the reports from the cores and determines which cores need to generate their path segments between which collision nodes. Note that some cores might have become superfluous, or may need to be linked in a non-sequential order (it can happen!)

- Phase 2: All relevant cores construct their local paths and report these back to the master core.

- The master core assembles the final path.

- All cores perform a final clean-up.

During phase 1, the essential cores basically perform a normal Fringe Search towards the way-points of their neighbor cores. Their searches start, of course, at either start node $S$ or goal node $G$, and not at the start or goal way-points of the high-level path. The search will continue until either a path can be constructed, or until there are no more nodes available which means we have to give up. Collisions with the 'nonessential' cores are analyzed to determine if a full path can be constructed, but they will not stop the cores. With some luck, we might be able to by-pass some nonessential cores, or maybe link up directly with the other essential core itself.

The 'non-essential' cores use a slightly different heuristic for cost estimation function. This function is initially biased to flood towards the local start nodes of the adjacent cores:

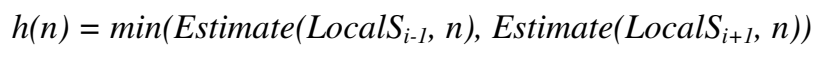

Estimate() is a cost estimation function, such as the Euclidean distance. As soon as a first collision with a direct neighbor has been detected, we switch to a normal heuristic that will only flood in the opposite direction, towards the other neighbor's local start node. Once we have collided with that one as well, but determined that a fullpath is not yet constructed, we just keep flooding with the original heuristic function again. This will make the flood boundary expand in all directions again which in turn might find other collisions that prove to be more beneficial. Only when a nonessential core runs out of nodes will it abort the search. This event does not explicitly have to be reported back to the master core in any way, it might just mean that our initial guess using the high-level path was 'wrong', and that the non-essential core started its search in an area that became isolated due to dynamic obstacles.

Once enough collisions have been detected and the master core has determined which cores will take part in the full path we can start phase 2. During this phase it is up to the corresponding cores to construct their local sections of the final full path. Synchronization between cores can all be done using spin-locks to ensure that there is no unintentional operating system overhead. Now, for essential cores it is very easy to construct their local path segments. As discussed earlier, the $\mathrm{A}^{*}$ algorithm keeps track of a 'parent node' for each node that it floods in order to link back towards its original start node. We thus only need to look-up the collision node and follow the parent links back to what will either be the original start node $S$ or goal node $G$. 


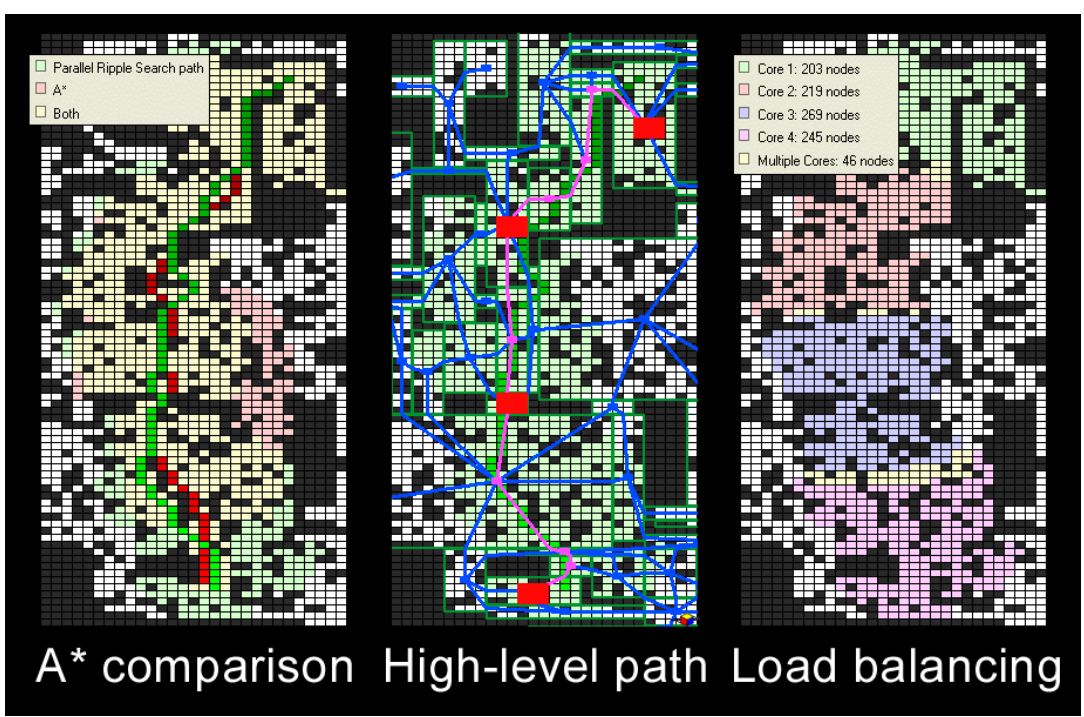

Fig. 5. Path generated with the PRS algorithm. (Left) comparison with the classic $A^{*}$. (Middle) The high-level path used, and the way-points used to start off the non-essential cores. (Right) Each core has flooded roughly the same amount of nodes. The yellow nodes indicated collisions.

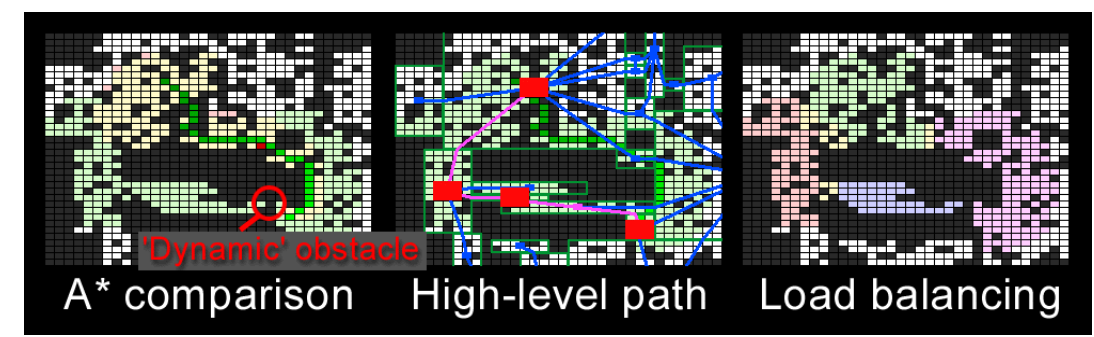

Fig. 6. Example of how the PRS algorithm still manages to bypass unexpected obstacles on the high-level path (middle). In this case, two cores did not manage to collide with each other (right) and thus only the other cores contributed to the resulting path. A comparison with the classic A* implementation (left) shows that both paths were virtually identical.

For non-essential cores we need to employ a different approach. We cannot use the parent links because these will always lead us back to the local start node at the location of the corresponding high-level path anchor node. As discussed previously for PHS, we need a smoothing phase in an attempt to 'iron out' the potential outlier that is the high-level path node itself. Especially if dynamic obstacles blocked our way, we need to make sure that the high-level path will not pull the resulting path way off course. For this, we do a new pathfinding session in order to find a suitable path between the two collision nodes that will ultimately 'bridge the gap' between the neighbors of the core. We can, however, significantly speed up this process by simply limiting the flood area to nodes that have been flooded before. This information is often directly available via 'visited flags' in the nodes themselves and thus the only 
extra cost is the pathfinding session itself. Note that, because the flood areas will ideally be relatively small, a large amount of data will already be in the core's cache, thus making such re-visits a very fast process. Once all local path segments have been obtained, the master core simply needs to copy them into a single buffer, making sure that no duplicate entries from the collision nodes are copied.

The overall implementation time of PRS was significantly longer compared to PBS, but not as long as that of DFS, provided that we already have the means for generating high-level paths. PRS requires more synchronization moments, and we do need to keep track on how to safely access memory without having race conditions causing real problems. The cores always share one memory pool in which they will write their unique core IDs for each node they flood. In this way, other cores can detect when they trod on each other toes and handle collisions. If we limit the size of the IDs to a single byte we can be sure enough that writing them is 'atomic' and the chance on race conditions is actually very small. And even then, if this does happen, the cores are bound to detect the collision during their next iterations as they further flood into each others 'body mass'.

Figure 5 shows an example of a path obtained with the PRS algorithm, 60\% faster than a classic $A^{*}$. The differences between both paths are small, mainly due to the fact that the high-level path has managed to make a very good 'guess'. PRS is also more robust and can much better handle unexpected obstacles on the high-level path, as shown in Figure 6. The quality of the paths is in general good, although they are still influenced by the orientation of the high-level path.

\subsection{Performance}

We have repeated for PRS the experimental measurements described in Section 3. As PBS was clearly the best alternative so far, we will limit the discussion here to Fringe Search, PBS and PRS in order to keep a clear overview.

From Figure 7 we conclude that PBS is still a very strong candidate on short to medium path lengths. Above roughly 200 nodes, PRS finally starts to capitalize on the fact that it can utilize more than two cores, at which the speed-up factor is in the range 2,5-10 compared to classic A*. Up to that point, PRS has too much overhead and/or cannot utilize all its cores when not enough way-points are found in the high-level graph. Regarding path quality, we can see that PRS generates paths that are on average about $4 \%$ more expensive. This deviation is partially due to the fact that the algorithm relies on the collisions to be favorable (which is, of course, not always the case), and also on the Fringe Search threshold relaxation, an 'artificial increment' in the FS's $f(n)$ threshold for opening new nodes, so that it expands faster outwards; see [4]. Also, in contrast to classic $\mathrm{A}^{*}$, we do not explicitly search for better nodes around collision nodes. The cost overhead for PBS is very low, less than $1 \%$ on average, which makes it an excellent alternative for short to medium length paths. The fact that PRS seems to have a 'constant' overhead factor indicates that, although the algorithm is 'probabilistic' in nature, it is still able to make good enough 'guesses' on a consistent basis. This is directly linked to the quality of the high-level graphs, which is therefore an essential component of any successful PRS implementation. 

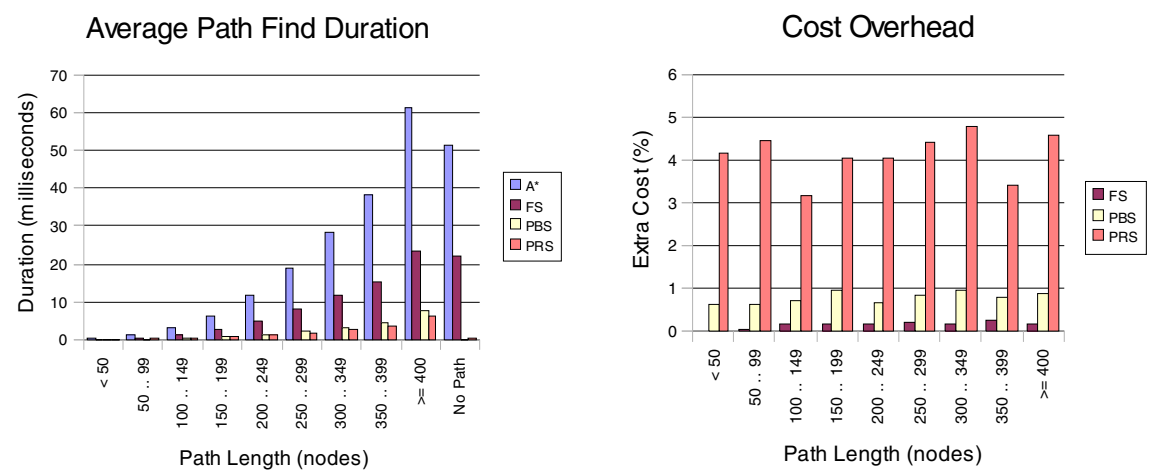

Fig. 7. (Left) Comparison of average pathfinding duration. (Right) Comparison of average cost overhead. The cost overhead remains fairly constant for all variants.

\section{Conclusion and Future Work}

We have implemented a number of parallel pathfinding algorithms in order to investigate their behavior and performance in multi-core architectures. We concluded that all these algorithms exhibit one or more weaknesses, for example, they either have a large overhead, yield far from optimal paths, do not easily scale up to many cores, or are cache unfriendly. The latter was found to be crucial, as currently available multicore CPUs have good cache look-ahead prediction, as long as shared pages do not get written into too often. In other words, data separation is key to efficient pathfinding for these architectures.

In this paper we proposed Parallel Ripple Search (PRS), a novel parallel pathfinding algorithm that largely solves the above limitations. Basically, the algorithm employs (i) two 'essential cores' to flood at the path extremities (like Parallel Bidirectional Search does), and (ii) all other available 'non-essential cores' to flood local search areas, starting at 'equidistant' intervals on a high-level path. These cores then use A* flooding behavior to expand towards each other, yielding good 'guesstimate points' at border touch on. As a result, all cores effectively run at full speed until enough waypoints have been found.

Like most other parallel algorithms, PRS sacrifices some path quality for speed: it runs roughly 2,5 up to 10 times faster than a classic $A^{*}$ implementation, with only an average minor penalty of $4 \%$ in path cost. This inevitable loss of optimality justifies the use of the Fringe Search variant, which is instrumental to further improve performance by means of its threshold relaxation: not only is more work done in parallel, it also expands flood boundaries faster, resulting in earlier collisions.

The PRS algorithm does not rely on any expensive parallel programming synchronization locks or mutexes, but instead relies on the opportunistic use of node collisions among cooperating cores, exploiting the multi-core's shared memory architecture. As a result, PRS is easily portable to different platforms that provide Symmetric Multiprocessing architectures and/or embedded systems that do not provide concurrent programming primitives other than threads. 
Future research should focus on, at least, two directions. First, it would be worthwhile improving the quality of the high-level path, enabling PRS to make better guesstimates on where the non-essential cores should best start flooding from. Second, new performance gains should be achieved by further reducing cache collisions between cores, e.g. by re-arranging the memory location of nodes to better reflect their real-world topology.

In short, the PRS algorithm (i) is a fast and practical pathfinding solution for large and complex maps, (ii) it flexibly handles dynamic obstacle in a natural way, and (iii) it guarantees good scalability facing the increasing amount of cores of present day hardware.

\section{References}

1. Amato, N.: Randomized Motion Planning. Texas, USA (2004), http: //parasol.tamu. edu/ amato/Courses/padova04/lectures / L8.prms.paf

2. Björnsson, Y., Enzenberger, M., Holte, R., Schaeffer, J.: Fringe Search: Beating A* at Pathfinding on Game Maps. In: Proceedings of IEEE Symposium on Computational Intelligence and Games, Essex, pp. 125-132 (2005),

http://www.cs.ualberta.ca/ games/pathfind/publications / cig2005.pdf

3. Bleiweiss, A.: GPU Accelerated Pathfinding. In: Proceedings of Eurographics 2008 (2008)

4. Botea, A., Muller, M., Schaeffer, J.: Near Optimal Hierarchical Path-Finding (HPA*). Department of Computing Science, University of Alberta (2006)

5. Brand, S.: Efficient obstacle avoidance using autonomously generated navigation meshes. MSc Thesis, Delft University of Technology, The Netherlands (2009)

6. Buluç, A., Gilbert, J.R., Budak, C.: Solving Path Problems on the GPU. Parallel Computing (2009), doi:10.0106/j.parco.2009.12.002

7. Cohen, D., Dallas, M.: Implementation of Parallel Path Finding in a Shared Memory Architecture. Department of Computer Science Rensselaer Polytechnic Institute, Troy, NY (2010), http: //www.cs.rpi .edu/ dallam/Parallel.pdf

8. Cvetanovic, Z., Nofsinger, C.: Parallel Astar Search on Message-Passing Architectures. System Sciences. In: Proceedings of the Twenty-Third Annual Hawaii Conference, vol. 1, pp. 92-90 (1990)

9. Patel, A.J. (2004) Variations of A*. Amit's Game Programming Site, http://www.dc.fi.udc.es/lidia/mariano/demos/amits/ theory.stanford.edu/ amitp/gameprogramming/variations .html

10. Sniedovich, M.: Dijkstra's Algorithm. The University of Melbourne, Australia, http://www.ms. unimelb.edu.au/ moshe/620-261/ dijkstra/dijkstra.html

11. Nohra, J., Champandard, A.J.: The Secrets of Parallel Pathfinding on Modern Computer Hardware. In: Intel Software Network, Intel Corp. (2010)

12. Sturtevant, N.R., Geisberger, R.: A Comparison of High-Level Approaches for Speeding Up Pathfinding. In: Proceedings of the Conference on Artificial Intelligence and Interactive Digital Entertainment, AIIDE 2010 (2010) 